DOI: $10.17516 / 1998-2836-0246$

УДК 541.138: 547.484.451

\title{
Electrochemical Study of the Redox Properties of Levulinic Acid in Organic and Aqueous-Organic Solutions
}

\author{
Galina V. Burmakina*a, \\ Dmitry V. Zimonin ${ }^{\mathrm{a}, \mathrm{b}}$ and Anatoly I. Rubaylo ${ }^{\mathrm{a}, \mathrm{b}}$ \\ ${ }^{a}$ Institute of Chemistry and Chemical Technology SB RAS \\ Krasnoyarsk, Russian Federation \\ ${ }^{b}$ Siberian Federal University \\ Krasnoyarsk, Russian Federation
}

Received 24.06.2021, received in revised form 25.07.2021, accepted 17.08.2021

\begin{abstract}
The redox properties of levulinic acid (LA) were studied by electrochemical methods in acetonitrile, a mixture of acetonitrile with water, ethanol, acetone, and dimethyl sulfoxide. It was shown that the irreversible two-electron reduction of LA under the studied conditions leads to the formation of $\gamma$-valerolactone. This reduction potential depends on the solvent and shifted anodically by following order: dimethyl sulfoxide $<$ acetonitrile $<$ acetone $<$ ethanol.
\end{abstract}

Keywords: electrochemistry, redox properties, levulinic acid, $\gamma$-valerolactone.

Citation: Burmakina, G. V., Zimonin, D. V., Rubaylo, A. I. Electrochemical study of the redox properties of levulinic acid in organic and aqueous-organic solutions, J. Sib. Fed. Univ. Chem., 2021, 14(3), 388-395. DOI: 10.17516/1998-2836-0246

(C) Siberian Federal University. All rights reserved

This work is licensed under a Creative Commons Attribution-NonCommercial 4.0 International License (CC BY-NC 4.0).

* Corresponding author E-mail address: bgvicct@gmail.com,bgv@akadem.ru 


\title{
Электрохимическое изучение редокс-свойств \\ левулиновой кислоты в органических \\ и водно-органических средах
}

\author{
Г.В. Бурмакина ${ }^{a}$, \\ Д. В. Зимонин ${ }^{\mathrm{a}, \boldsymbol{\sigma}}$, А.И. Рубайло \\ ${ }^{a}$ Институт химии и химической технологии \\ ФИЦ КНЦ СО РАН \\ Российская Федераџия, Красноярск \\ ${ }^{6}$ Сибирский федеральньий университет \\ Российская Федераџия, Красноярск
}

\begin{abstract}
Аннотация. Редокс-свойства левулиновой кислоты (ЛК) в ацетонитриле, смеси ацетонитрила с водой, этаноле, ацетоне и диметилсульфоксиде исследованы электрохимическими методами. Показано, что ЛК в изученных условиях восстанавливается с присоединением двух электронов и образованием $\gamma$-валеролактона. Значение потенциала восстановления ЛК зависит от природы растворителя и смещается в анодную область в ряду: диметилсульфоксид < ацетонитрил < ацетон < этанол,
\end{abstract}

Ключевые слова: электрохимия, редокс-свойства, левулиновая кислота, $\gamma$-валеролактон.

Цитирование: Бурмакина, Г.В. Электрохимическое изучение редокс-свойств левулиновой кислоты в органических и водно-органических средах / Г. В. Бурмакина, Д. В. Зимонин, А. И. Рубайло // Журн. Сиб. федер. ун-та. Химия, 2021, 14(3). C. 388-395. DOI: $10.17516 / 1998-2836-0246$

\section{Введение}

Левулиновая кислота (ЛК), получаемая в результате гидролиза лигноцеллюлозной биомассы, является одной из универсальных молекул-«платформ» для синтеза ценных химических веществ и высокоэнергетических жидких топлив [1-3]. Наличие реакционноспособных кето- и карбоксильной функциональных групп в молекуле, их взаиморасположение обеспечивают многообразие синтетических превращений ЛК [4]. Множество публикаций посвящено исследованию гомогенных и гетерогенных каталитических процессов превращений левулиновой кислоты [5-13], в то время как работ по электрохимической конверсии ЛК незначительно. При этом большинство исследований электрохимических реакций с участием ЛК проведено в водных растворах [14-17]. Однако использование органических растворителей позволяет проводить электрохимические исследования соединений в более широкой области потенциалов и, следовательно, более детально изучать механизмы редокс-превращений органических веществ в различных процессах.

Поэтому цель настоящей работы - электрохимическое изучение редокс-свойств левулиновой кислоты в различных органических и водно-органических средах: ацетонитриле, смеси ацетонитрила с водой, этаноле, ацетоне, диметилсульфоксиде.

$$
-389-
$$




\section{Экспериментальная часть}

Электрохимические измерения свежеприготовленных растворов левулиновой (Sigma Aldrich) и валериановой (для хроматографии, 99 \%) кислот, $\gamma$-валеролактона (ГВЛ) (Sigma Aldrich) проводили в атмосфере аргона при комнатной температуре. В качестве органических растворителей использовали ацетонитрил $\left(\mathrm{CH}_{3} \mathrm{CN}\right.$, осч), этанол $\left(\mathrm{C}_{2} \mathrm{H}_{5} \mathrm{OH}\right.$, чда, 96 \%), ацетон $\left(\mathrm{CH}_{3} \mathrm{COCH}_{3}\right.$, осч), диметилсульфоксид (ДМСО, $\left(\mathrm{CH}_{3}\right)_{2} \mathrm{SO}$, чда); фоновый электролит - $0.1 \mathrm{M}$ тетраэтиламмоний тетрафторборат $\left[\left(\mathrm{C}_{2} \mathrm{H}_{5}\right)_{4} \mathrm{NBF}_{4}\right]$. Водные и водно-органические растворы готовили с применением деионизованной воды, полученной при помощи системы очистки воды Direct-Q 3 (Millipore, France) с электропроводностью менее $10^{-7} \mathrm{OM}^{-1} \mathrm{~cm}^{-1}$. Циклические вольтамперограммы (ЦВА) регистрировали на потенциостате IPC-Pro М (ООО НТФ «Вольта», СанктПетербург, Россия). В качестве стационарных твердых рабочих электродов ${ }^{1}$ использовали платиновый $(\mathrm{d}=1 \mathrm{mм})$ или стеклоуглеродный $(\mathrm{CУ})(\mathrm{d}=5$ мм) электрод в тефлоновых корпусах (d = 10 мм). Электродом сравнения служил полуэлемент $\mathrm{Ag} / 0.1 \mathrm{M} \mathrm{AgNO}_{3}$ в $\mathrm{CH}_{3} \mathrm{CN}$ или платиновая спираль, соединенные с ячейкой электролитическим мостом, заполненным фоновым электролитом, через капилляр Луггина, вспомогательным - платиновая спираль, помещенная в стеклянную трубку с пористым фильтром. Число электронов, участвующих в каждой редоксстадии, определяли сравнением высот волн исследуемых соединений с высотой волны обратимого одноэлектронного редокс-перехода ферроцен-ферроцений.

\section{Результаты и обсуждение}

Проведено сравнительное исследование электрохимического поведения левулиновой кислоты в 4 мМ водном растворе $\mathrm{H}_{2} \mathrm{SO}_{4}$ и в смеси ацетонитрила с водой (1:1) на Рt-электроде. Циклические вольтамперограммы левулиновой кислоты в изученных условиях приведены на рис. 1.

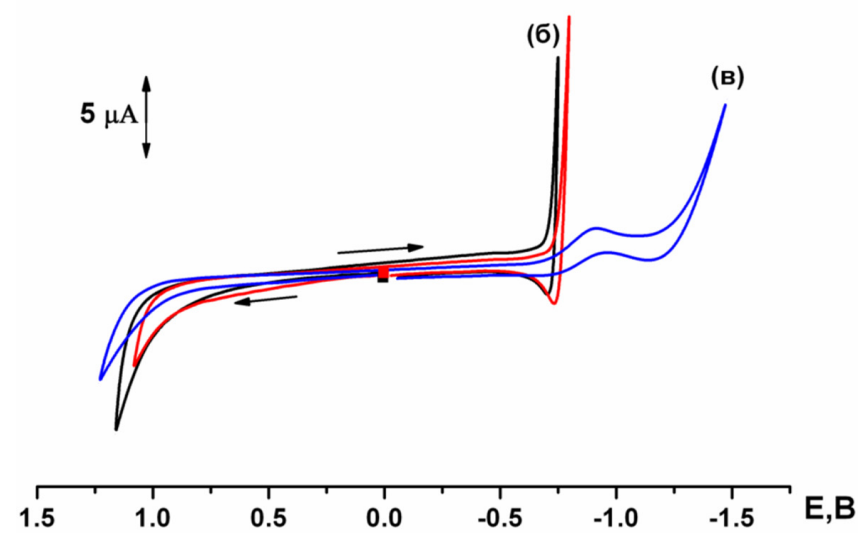

Рис. 1. ЦВА: (a) 4 мМ водный раствор $\mathrm{H}_{2} \mathrm{SO}_{4}$; (б) ЛК в 4 мМ водном растворе $\mathrm{H}_{2} \mathrm{SO}_{4}$, (в) ЛК в $\mathrm{CH}_{3} \mathrm{CN}+\mathrm{H}_{2} \mathrm{O}$ (1:1) $0.1 \mathrm{M}\left(\mathrm{C}_{2} \mathrm{H}_{5}\right)_{4} \mathrm{NBF}_{4}(\mathrm{Pt}, \mathrm{C}=1 \mathrm{mM}, \mathrm{V}=25 \mathrm{mB} / \mathrm{c}$, отн. $\mathrm{Pt})$

Fig. 1. Cyclic voltammograms of (a) $4 \mathrm{mM} \mathrm{H}_{2} \mathrm{SO}_{4}$ aqueous solution; (б) $\mathrm{LA}$ in $4 \mathrm{mM} \mathrm{H}_{2} \mathrm{SO}_{4}$ aqueous solution; (b) $\mathrm{LA}$ in $\mathrm{CH}_{3} \mathrm{CN}+\mathrm{H}_{2} \mathrm{O}(1: 1) 0.1 \mathrm{M}\left(\mathrm{C}_{2} \mathrm{H}_{5}\right)_{4} \mathrm{NBF}_{4}(\mathrm{Pt}, \mathrm{C}=1 \mathrm{mM}, \mathrm{V}=25 \mathrm{mV} / \mathrm{s}$, vs Pt)

Использование различных рабочих электродов позволяет исследовать процессы окисления и восстановления соединений в более широкой области потенциалов. Так, рабочая область измерения потенциалов в ацетонитриле (относительно $\mathrm{Ag} / 0.1 \mathrm{M} \mathrm{AgNO}_{3}$ в MeCN) на Pt- и СУ-электродах от 2.00 до-2.20 В и от 2.00 до-2.60 В соответственно. 
Как видно из рис. 1, волна восстановления левулиновой кислоты в 4 мМ водном раствоpe $\mathrm{H}_{2} \mathrm{SO}_{4}$ практически сливается с волной восстановления фонового электролита, а именно восстановления протонов (рис. $1 a, \sigma)$, в то время как в водно-ацетонитрильном (1:1) растворе наблюдается хорошо определяемая двухэлектронная волна восстановления ЛК (рис. 1 в). Полученные результаты свидетельствуют о том, что использование органических растворителей, например, таких как ацетонитрил, позволяет более качественно определять электрохимические характеристики органических веществ: количество стадий, значение потенциала восстановления и количество электронов, участвующих в электродной реакции.

В работе редокс-свойства левулиновой кислоты, а также возможных продуктов ее восстановления - валериановой кислоты и $\gamma$-валеролактона, изучены методами циклической вольтамперометрии на Pt- и СУ-электродах в безводном ацетонитриле, смеси ацетонитрила с водой (1:1), этаноле, ацетоне, диметилсульфоксиде. Установлено, что $\gamma$-валеролактон электрохимически не активен в изученных условиях. Электрохимические характеристики восстановления левулиновой и валериановой кислот приведены в таблице, ЦВА левулиновой кислоты на Ptэлектроде в различных растворителях - на рис. 2 .

Как видно из данных таблицы и рис. 2, левулиновая кислота в изученных органических растворителях, кроме ДМСО, восстанавливается на Pt- и СУ-электродах в одну двухэлектронную необратимую стадию. В ДМСО наблюдается разделение двухэлектронной волны восстановления ЛК на две одноэлектронные (рис. 22). Значения потенциалов восстановления ЛК также зависят от природы растворителя и смещаются в анодную область в ряду: диметил-

Таблица. Электрохимические характеристики восстановления левулиновой и валериановой кислот Table. Electrochemical characteristics for the reduction of levulinic and valeric acids

\begin{tabular}{|c|c|c|}
\hline \multirow{2}{*}{ Условия эксперимента } & \multicolumn{2}{|c|}{$\mathrm{E}_{1 / 2}, \mathrm{~B}(\mathrm{n})$} \\
\hline & $\mathrm{Pt}$ & CY \\
\hline \multicolumn{3}{|c|}{ Левулиновая кислота } \\
\hline $\mathrm{CH}_{3} \mathrm{CN}, 0.1 \mathrm{M}\left(\mathrm{C}_{2} \mathrm{H}_{5}\right)_{4} \mathrm{NBF}_{4}$, отн. $\mathrm{Ag} / 0.1 \mathrm{M} \mathrm{Ag}^{+}$ & $-1,26(2)$ & $-2,24(2)$ \\
\hline $\mathrm{CH}_{3} \mathrm{CN}, 0.1 \mathrm{M}\left(\mathrm{C}_{2} \mathrm{H}_{5}\right)_{4} \mathrm{NBF}_{4}$, отн. Pt & $-1,20(2)$ & $-2,22(2)$ \\
\hline $\mathrm{CH}_{3} \mathrm{CN}+\mathrm{H}_{2} \mathrm{O}(1: 1), 0.1 \mathrm{M}\left(\mathrm{C}_{2} \mathrm{H}_{5}\right)_{4} \mathrm{NBF}_{4}$, отн. $\mathrm{Pt}$ & $-0,82(2)$ & $-1,75(2)$ \\
\hline $\mathrm{C}_{2} \mathrm{H}_{5} \mathrm{OH}, 0.1 \mathrm{M}\left(\mathrm{C}_{2} \mathrm{H}_{5}\right)_{4} \mathrm{NBF}_{4}$, отн. $\mathrm{Pt}$ & $-0,99(2)$ & - \\
\hline $\mathrm{CH}_{3} \mathrm{COCH}_{3}, 0.1 \mathrm{M}\left(\mathrm{C}_{2} \mathrm{H}_{5}\right)_{4} \mathrm{NBF}_{4}$, отн. $\mathrm{Pt}$ & $-1,16(2)$ & $-2,19(2)$ \\
\hline$\left(\mathrm{CH}_{3}\right)_{2} \mathrm{SO}, 0.1 \mathrm{M}\left(\mathrm{C}_{2} \mathrm{H}_{5}\right)_{4} \mathrm{NBF}_{4}$, отн. Pt & $\begin{array}{c}-1,25(2) \\
{[-1,19(1)} \\
-1,34(1)]\end{array}$ & $-2,46(2)$ \\
\hline \multicolumn{3}{|c|}{ Валерьяновая кислота } \\
\hline $\mathrm{CH}_{3} \mathrm{CN}, 0.1 \mathrm{M}\left(\mathrm{C}_{2} \mathrm{H}_{5}\right)_{4} \mathrm{NBF}_{4}$, отн. Pt & $\begin{array}{c}-1,35(2) \\
-1,72(<1)\end{array}$ & $-2,24(2)$ \\
\hline $\mathrm{CH}_{3} \mathrm{CN}+\mathrm{H}_{2} \mathrm{O}(1: 1), 0.1 \mathrm{M}\left(\mathrm{C}_{2} \mathrm{H}_{5}\right)_{4} \mathrm{NBF}_{4}$, отн. $\mathrm{Pt}$ & $-0,94(1)$ & - \\
\hline
\end{tabular}

Примечание: $\mathrm{n}$ - число электронов, участвующих в электрохимической стадии (знак «<» означает, что высота волны исследуемого соединения меньше, чем высота одноэлектронной волны).

Notes: $\mathrm{n}$ - the number of electrons transferred in a particular electrochemical stage (the sign $«<»$ indicates that the wave height is smaller than the height of the one-electron wave). 


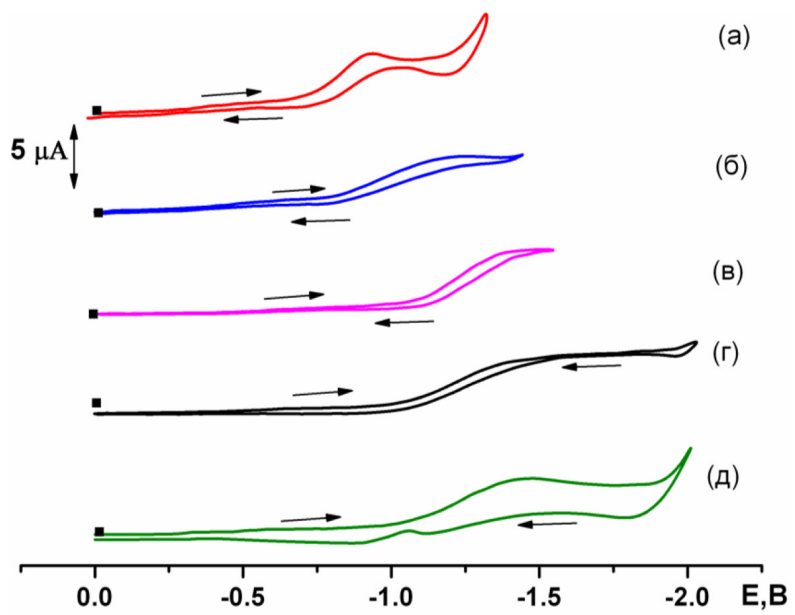

Рис. 2. ЦВА ЛК в различных растворителях: (a) ацетонитрил + вода (1:1); (б) этанол; (в) ацетон; (2) ацетонитрил; (д) ДМСО (Pt, $0.1 \mathrm{M}\left(\mathrm{C}_{2} \mathrm{H}_{5}\right)_{4} \mathrm{NBF}_{4}, \mathrm{C}=2 \mathrm{MM}, \mathrm{V}=25 \mathrm{mB} / \mathrm{c}$, отн. $\left.\mathrm{Pt}\right)$

Fig. 2. Cyclic voltammograms of the LA in various solvents: $(a)$ acetonitrile + water $(1: 1)(\sigma)$ ethanol (b) acetone (2) acetonitrile, (d) DMSO (Pt, $0.1 \mathrm{M}\left(\mathrm{C}_{2} \mathrm{H}_{5}\right)_{4} \mathrm{NBF}_{4}, \mathrm{C}=2 \mathrm{mM}, \mathrm{V}=25 \mathrm{mV} / \mathrm{s}$, vs Pt)<smiles>CC(=O)CCC(=O)O</smiles>

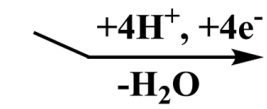

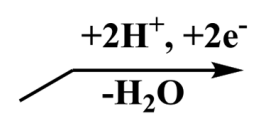<smiles>CC1CCC(=O)O1</smiles>

путь 1

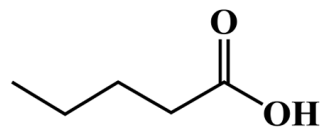

путь 2

Рис. 3. Пути электрохимического восстановления левулиновой кислоты

Fig. 3. Reaction pathways of the electrochemical reduction of levulinic acid

сульфоксид < ацетонитрил < ацетон < этанол (табл., рис. 2). Добавление воды в ацетонитрил (1:1) приводит к значительному смещению значения $\mathrm{E}_{1 / 2}$ двухэлектронного восстановления ЛК в анодную область (табл., рис. $2 a$, г) и уменьшению диапазона рабочей области потенциалов в водно-ацетонитрильном растворе от 1,2 до -1,5 В (рис. 18) по сравнению с безводным ацетонитрилом (от 2,00 до -2,20 В). Значения потенциалов восстановления ЛК зависят и от природы материала рабочего электрода - на СУ значительно смещаются в катодную область потенциалов по сравнению с восстановлением на Рt-электроде (табл.).

Известно $[14,16]$, что восстановление левулиновой кислоты в водных растворах может протекать по двум маршрутам: при $\mathrm{pH}=$ 7,5 с присоединением двух электронов, двух протонов и образованием $\gamma$-валеролактона, при $\mathrm{pH}=0$ наблюдается более глубокое четырехэлектронное восстановление ЛК до валериановой кислоты по схеме, представленной на рис. 3 :

Для установления схемы восстановления левулиновой кислоты в органических и водноорганических средах изучено ее электрохимическое поведение в присутствии тетрафтороборной кислоты $\left(\mathrm{HBF}_{4}\right.$ используется в качестве доноров протонов в органических растворителях с фоновым электролитом, содержащим анион $\left.\left[\mathrm{BF}_{4}\right]^{-}\right)$. На рис. 4 приведены ЦВА левулиновой 


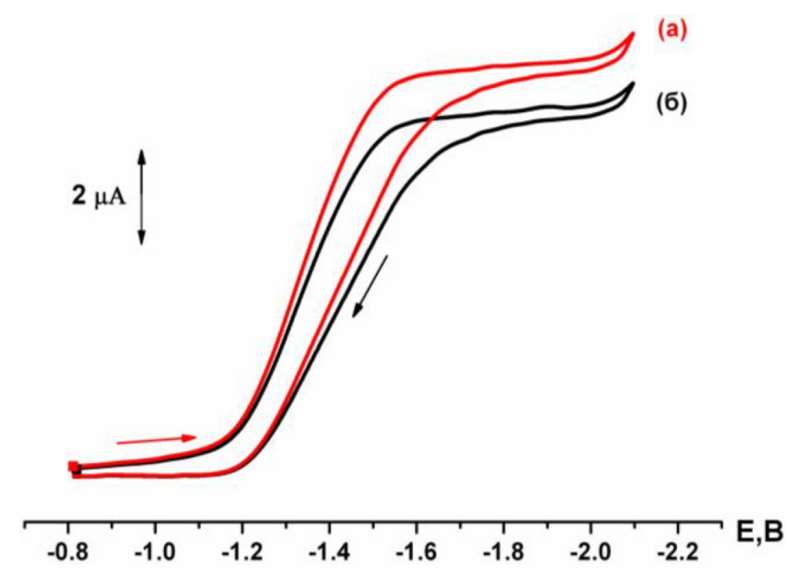

Рис. 4. ЦВА: (a) ЛК с добавлением $\mathrm{HBF}_{4}(1: 1)$; (б) ЛК (Pt, $\mathrm{CH}_{3} \mathrm{CN}, 0.1 \mathrm{M}\left(\mathrm{C}_{2} \mathrm{H}_{5}\right)_{4} \mathrm{NBF}_{4}, 2 \mathrm{мM}, \mathrm{V}=25$ мB/c, отн. Pt)

Fig. 4. Cyclic voltammograms of (a) LA with the addition of $\mathrm{HBF}_{4}(1: 1)$, (б) LA ( $\mathrm{Pt}, \mathrm{CH}_{3} \mathrm{CN}, 0.1 \mathrm{M}\left(\mathrm{C}_{2} \mathrm{H}_{5}\right)_{4} \mathrm{NBF}_{4}$, $2 \mathrm{mM}, \mathrm{V}=25 \mathrm{mV} / \mathrm{s}$, vs Pt)

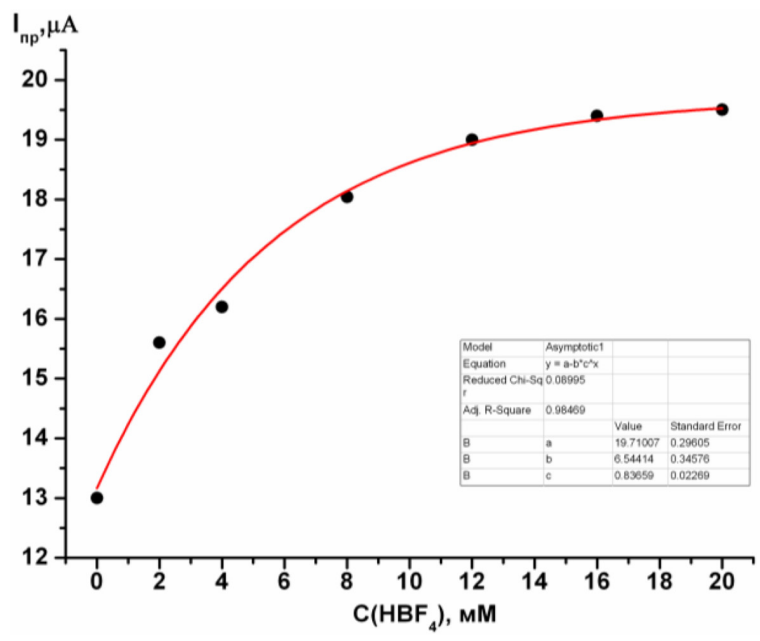

Рис. 5. График зависимости предельного тока волны восстановления ЛК $\left(\mathrm{I}_{\text {пр }}\right)$ от концентрации $\mathrm{HBF}_{4}$ $\left(\mathrm{C}_{\text {ЛК }}=2 \mathrm{MM}\right)$

Fig. 5. Plot of limiting current of LA reduction versus the concentration of $\mathrm{HBF}_{4}\left(\mathrm{C}_{\mathrm{LA}}=2 \mathrm{mM}\right)$

кислоты в отсутствие и с добавлением $\mathrm{HBF}_{4}$, график зависимости предельного тока волны восстановления ЛК ( $\mathrm{I}_{\text {пр }}$ ) от концентрации $\mathrm{HBF}_{4}$ - на рис. 5.

Как видно из рис. 4, на ЦВА левулиновой кислоты при добавлении к ее раствору $\mathrm{HBF}_{4}$ (в соотношении 1:1) наблюдается увеличение высоты волны восстановления ЛК (рис. 4a) по сравнению с ее восстановлением без $\mathrm{HBF}_{4}$ (рис. 4б), что свидетельствует об участии протона при восстановлении левулиновой кислоты. Дальнейшее добавление $\mathrm{HBF}_{4}$ к раствору ЛК (до соотношения 1:10) приводит к постепенному увеличению высоты ее волны с последующим замедлением, стремящимся к соотношению высот волн ЛК и ЛК $+\mathrm{HBF}_{4}$ примерно 2 к 3 , т. е. 2 $\mathrm{H}^{+}$на три молекулы ЛК. 
Таким образом, полученные в работе результаты свидетельствуют о том, что в изученных органических и водно-органических средах с $\mathrm{pH}$, близким к нейтральному, электрохимическое восстановление левулиновой кислоты протекает с присоединением двух электронов и образованием $\gamma$-валеролактона.

\section{Благодарность / Acknowledgements}

Работа выполнена в рамках государственного задания Института химии и химической технологии СО РАН (проект 0287-2021-0012).

This work was conducted within the framework of the budget project 0287-2021-001 for Institute of Chemistry and Chemical Technology SB RAS.

\section{Список литературы / References}

1. Bozell J.J., Moens L., Elliott D.C., Wang Y., Neuenscwander G. G., Fitzpatrick S. W., Bilski R. J., Jarnefeld J.L. Production of levulinic acid and use as a platform chemical for derived products. Resources, Conservation and Recycling 2000. Vol. 28(3-4), P. 227-239.

2. Bozell J. J., Petersen G. R. Technology development for the production of biobased products from biorefinery carbohydrates - the US Department of Energy's «Top 10» revisited. Green Chem. 2010. Vol. 12, P. 539-554.

3. Serrano-Ruiz J.C., Wang D., Dumesic J. A. Catalytic upgrading of levulinic acid to 5-nonanone. Green Chem. 2010. Vol. 12, P. 574-577.

4. Timokhin B. V., Baransky V. A., Eliseeva G. D. Levulinic acid synthesis. Rus. Chem. Rev. 1999. Vol. 68(1), P. 73-84.

5. Yun, W.-C. Yang M.-T., Lin K.-Y.A. Water-born Zirconium-based metal organic frameworks as green and effective catalysts for catalytic transfer hydrogenation of levulinic acid to $\gamma$-valerolactone: critical roles of modulators. J. Col. Int.Sci. 2019. Vol. 543, P. 52-63.

6. Gundeboina R., Gadasandula S., Velisoju V.K., Gutta N., Kotha L.R., Aytam H.P. Ni-Al-Ti hydrotalcite based catalyst for the selective hydrogenation of biomass-derived levulinic acid to $\gamma$ valerolactone. Chem. Sel. 2019. Vol. 4(1), P. 202-210.

7. Yun W.-C., Lin T.-Y., Chiu H.-Y., Lin K.-Y.A. Microwave irradiation-enhanced catalytic transfer hydrogenation of levulinic acid to $\gamma$-valerolactone using Ruthenium: a comparative study with conventional heating processes. Waste and Biomass Valorization 2019. P. 1-11.

8. Mitta H., Seelam P.K., Raghava Chary K.V., Mutyala S., Boddula R., Inamuddin, Asiri A.M. Efficient vapor-phase selective hydrogenolysis of bio-levulinic acid to $\gamma$-valerolactone using $\mathrm{Cu}$ supported on hydrotalcite catalysts. Global Challenges 2018. Vol. 2(12), № 1800028. P. 1-12.

9. Gundekari S., Srinivasan K. Hydrous ruthenium oxide: A new generation remarkable catalyst precursor for energy efficient and sustainable production of $\gamma$-valerolactone from levulinic acid in aqueous medium. Appl. Cat. A: Gen. 2019. Vol. 569, P. 117-125.

10. Li S., Wang Y., Yang Y., Chen B., Tai J., Liu H., Han B. Conversion of levulinic acid to $\gamma$ valerolactone over ultra-thin $\mathrm{TiO}_{2}$ nanosheets decorated with ultrasmall $\mathrm{Ru}$ nanoparticle catalysts under mild condition. Green Chem. 2019. Vol. 21(4), P. 770-774.

11. Hirayama J., Orlowski I., Iqbal S., Douthwaite M., Ishikawa S., Miedziak P. J., Bartley J.K., Edwards J., He Q., Jenkins R.L., Murayama T., Reece C., Ueda W., Willock D. J., Hutchings G. J. The 
effects of dopants on the $\mathrm{Cu}-\mathrm{ZrO}_{2}$ catalysed hydrogenation of levulinic acid. J. Phys. Chem. C. 2019. Vol. 123(13), P. 7879-7888.

12. Mallesham B., Sudarsanam P., Reddy B. V.S., Rao B. G., Reddy B. M. Nanostructured Nickel/ Silica catalysts for continuous flow conversion of levulinic acid to $\gamma$-valerolactone. ACS Omega 2018. Vol. 3(12), P. 16839-16849.

13. Li F., Li Z., France L. J., Mu J., Song C., Chen Y., Jiang L., Long J., Li X. Highly efficient transfer hydrogenation of levulinate esters to $\gamma$-valerolactone over basic Zirconium carbonate. Ind. Eng. Chem. Res. 2018. Vol. 57(31), P. 10126-10136.

14. Xin L., Zhang Z., Qi J., Chadderdon D. J., Qiu Y., Warsko K. M., Li W. Electricity storage in biofuels: selective electrocatalytic reduction of levulinic acid to valeric Acid acid or $\gamma$-valerolactone. ChemSusChem 2013. Vol. 6, P. 674-686.

15. Nilges P., Santos T.R., Harnisch F., Schroder U. Electrochemistry for biofuel generation: electrochemical conversion of levulinic acid to octane. Energy Environ. Sci. 2012. Vol. 5, P. 5231-5235.

16. Santos T.R., Nilges P., Sauter W., Harnischb F., Schroder U. Electrochemistry for the generation of renewable chemicals: electrochemical conversion of levulinic acid. RSC Adv. 2015. Vol. 5, P. 26634-26643.

17. Qiu Y., Xin L., Chadderdon D. J., Qi J., Liang C., Li W. Integrated electrocatalytic processing of levulinic acid and formic acid to produce biofuel intermediate valeric acid. Green Chem. 2014. Vol. 16, P. 1305-1315. 